\title{
The Low-Frequency Array (LOFAR): Opening a New Window on the Universe
}

\author{
Namir E. Kassim, T. Joseph W. Lazio \\ Naval Research Laboratory, Code 7213, Washington, DC 20375-5351 \\ $U S A$ \\ William C. Erickson \\ Bruny .Island Radio Observatory, 42 Lighthouse Rd., Bruny Island, \\ Tasmania 7150 Australia \\ Patrick C. Crane \\ Interferometrics Inc., Chantilly, VA 20151 USA \\ R. A. Perley \\ National Radio Astronomy Observatory, P.O. Box 0, Socorro, NM \\ 87801 USA \\ B. Hicks \\ Naval Research Laboratory, Code 7218, Washington, DC 20375-5351 \\ $U S A$
}

\begin{abstract}
Decametric wavelength imaging has been largely neglected in the quest for higher angular resolution because ionospheric structure limited interferometric imaging to short $(<5 \mathrm{~km})$ baselines. The long wavelength ( $\mathrm{LW}, 2-20 \mathrm{~m}$ or $15-150 \mathrm{MHz}$ ) portion of the electromagnetic spectrum thus remains poorly explored. The NRL-NRAO $74 \mathrm{MHz}$ Very Large Array has demonstrated that self-calibration techniques can remove ionospheric distortions over arbitrarily long baselines. This has inspired the Low Frequency Array (LOFAR) - a fully electronic, broad-band (15$150 \mathrm{MHz}$ )antenna array which will provide an improvement of 2-3 orders of magnitude in resolution and sensitivity over the state of the art.
\end{abstract}

\section{Introduction}

The poor angular resolution arising from the ionospheric structure limit to short $(<5 \mathrm{~km}$ ) baselines at long wavelengths (LW, 2-20 m or $15-150 \mathrm{MHz}$ ) has left the LW region among the most poorly explored in the entire spectrum. Thus the demonstration by the $74 \mathrm{MHz}$ VLA (Kassim \& et al. (1993)), that self-calibration can remove ionospheric distortions over long baselines, now affords the opportunity to open a new high-resolution, high-sensitivity window on the electromagnetic spectrum at the longest wavelengths (Kassim \& Erickson (1998)). 
The jointly developed NRL-NRAO $74 \mathrm{MHz}$ observing system has attracted a variety of innovative proposals. Figure 1 provides an example of the types of images being produced by this system. The wide field of view $\left(>10^{\circ}\right)$, angular resolution $\left(\sim 1^{\prime}\right)$, and sensitivity $\left(\approx 25 \mathrm{mJy}^{\text {beam }}{ }^{-1}\right)$ provided by this single pointing demonstrate the unique imaging power offered by LW systems.

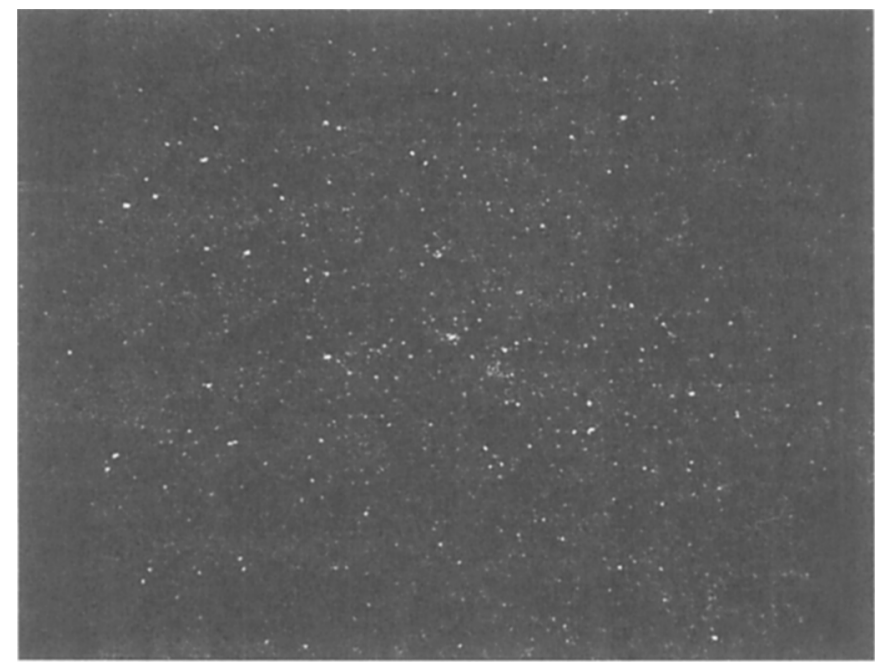

Figure 1. The Coma cluster of galaxies at $74 \mathrm{MHz}$ (Kronberg (1999)). The rms noise level is $25 \mathrm{mJy} \mathrm{beam}^{-1}$, and the field covers approximately $15^{\circ}$ at a resolution of $1^{\prime}$.

The VLA system (Kassim \& et al. (1993)), whose unprecedented angular resolution $\left(\approx 20^{\prime \prime}\right)$ and sensitivity $\left(\approx 30 \mathrm{mJy}\right.$ beam $\left.{ }^{-1}\right)$ despite modest collecting area $\left(\sim 10^{3} \mathrm{~m}^{2}\right)$, provide strong incentives to develop a much larger ( $\sim 500 \mathrm{~km}$ baselines) and more sensitive $\left(A_{\text {eff }}>10^{6} \mathrm{~m}^{2}\right)$ instrument. A completely electronic Low Frequency Array (LOFAR) could explore the entire LW spectrum at unmatched levels of sensitivity (sub-mJy) and angular resolution (arc-second)(Kassim \& Erickson (1998)). Such an instrument will provide a unique and efficient tool both for large-scale emission studies (e.g., supernova remnants, the inner Galaxy, and galaxy and cluster halos) and for high sensitivity observations of small-diameter steep spectrum sources (e.g., pulsars and high redshift radio galaxies). Furthermore, LOFAR would be an ideal receiver for solar radar imaging of Earthward-bound coronal mass ejections (CMEs), opening a new field of space weather prediction.

In $\S 2$. we present the scientific justification for developing LOFAR. In $\S 3$. we outline the technical concept for LOFAR - a broad-band, entirely electronic telescope. We have an exciting opportunity for a detailed exploration of the wavelength regime where radio astronomy was first discovered. These and our other conclusions are summarized in $\S 4$. 


\section{Scientific Objectives of LOFAR}

In this section we highlight key science projects which could be carried out with LOFAR. We emphasize that this list is by no means complete. Other topics include studies of star forming galaxies, halo emission from galaxies, emission and absorption mechanisms in galaxies, the search for fossil radio galaxies, studies of supernova remnants and their connection to cosmic-ray acceleration, the physical parameters of $\mathrm{H}$ II regions, interstellar propagation effects, carbon recombination lines, and the search for coherent emission form extrasolar planets.

\subsection{Cluster Radio Halos and Intergalactic Magnetic Fields}

A small number of galaxy clusters show radio halos-diffuse, central radio emission not associated with any one galaxy in the cluster. Models for the origins of these halos include an injection of relativistic electrons from radio galaxies, turbulent in situ particle acceleration, or a merger shock wave and secondary production from hadronic interactions of cosmic ray protons with the intracluster medium (Schlickeiser \& et al.(1987)).

Most models predict a much higher number of cluster halos than observed. The "missing" halos may have escaped detection by virtue of their steep spectra, suggesting that a search at low frequencies will find many more. Many models also predict that radio halos are transient, and a pool of relic electrons with energies $E \sim 100 \mathrm{MeV}$ is expected due to a maximum of the cooling time in this energy range (Sarazin(1999)). Indirect evidence for the existence of these relic electrons has been found recently as excess extreme ultraviolet emission from clusters (Hwang(1997), Enßlin \& Biermann(1998), Sarazin \& Lieu(1998), Bowyer \& Berghöfer(1998)), and if if the radio synchrotron emission from these halos can be detected, the combined information will provide a measurement of the magnetic field strength inside the intracluster medium. The combination of the number density of halos and magnetic field strength within them will provide much more stringent observational constraints for models.

\subsection{Radio and Star Forming Galaxies}

Radio galaxies are a varied group of objects (cf. Figure 2), ranging from highluminosity objects extending over megaparsec scales and presumably powered by massive black holes to low-luminosity, relatively small objects powered by star formation. The combination of the high sensitivity and angular resolution of LOFAR could be brought to bear on a number of outstanding problems in the origin, evolution, and nature of radio galaxies.

The redshifts at which these objects are detected, for a standard cosmological model, imply that only 1 Gyr has elapsed since the Big Bang. This leaves little time for jet-producing central engines and host galaxies to form, and the detection of these objects provides stringent constraints for models of active galactic nuclei formation.

LOFAR's frequency range will enable it to play a key role in the identification of the most distant radio galaxies. The highest-redshift $(z>3)$ sources known to date are characterized by steep spectra $(\alpha<-1)$ and small angular sizes $\left(\lessgtr 30^{\prime \prime}\right)$, and low frequency observations have been key to detecting them. For example the $6 \mathrm{C}^{*}$ sample of steep-spectrum sources resulted from a cross- 


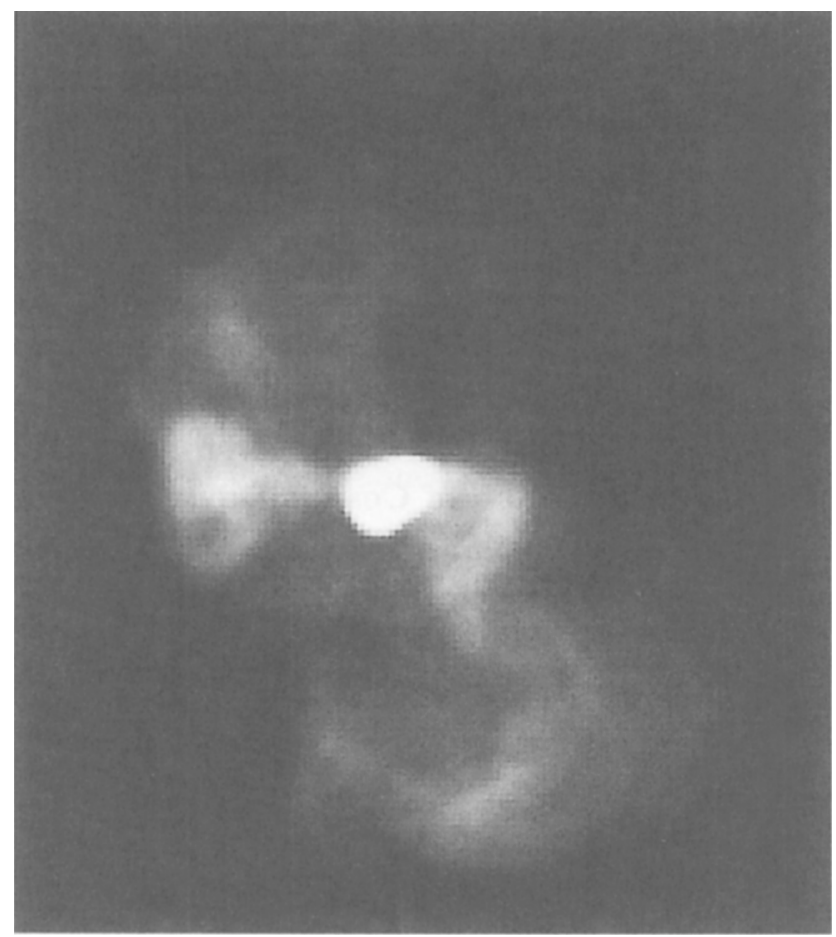

Figure 2. Virgo A at $74 \mathrm{MHz}$ (Kassim \& et al. (1993)). This image has an angular resolution of $25^{\prime \prime}$ and is approximately $15^{\prime}$ in size.

correlation of 151 and $5000 \mathrm{MHz}$ surveys (Blundell \& et al.(1998)), and led to the detection of $6 \mathrm{C}^{*} 0140+326$, among the most distant known radio galaxies $(z=4.4)$. The steep spectra of these sources ultimately limits the utility of a $151 \mathrm{MHz}$ survey; such a survey selects objects at a redshift $z$ according to their surface brightness at a rest-frame frequency of $(1+z) 151 \mathrm{MHz}$. In the case of an object at a redshift of 6 , this survey would select rest-frame emission near $1 \mathrm{GHz}$. Above $1 \mathrm{GHz}$, radio galaxy lobes are subject to synchrotron losses, and in addition their lobes suffer inverse Compton losses off the cosmic background radiation, whose energy density increases as $(1+z)^{4}$. LOFAR will lower the finding frequency at increased sensitivity, naturally finding more distant objects and generating a more complete sample of high-redshift radio galaxies.

Similar principles will also make LOFAR a powerful instrument for studying distant, star forming galaxies. Ground-based spectroscopy and the Hubble Deep Field suggest that star formation peaked at a redshift $z=1-2$ (Madau(1997)). However, recent observations at sub-millimeter wavelengths (e.g., with SCUBA) are revealing what appears to be a population of extremely dust-obscured, active star forming galaxies at high redshift (Smail \& et al.(1997), Barger \& et al. 
(1998), Hughes \& et al.(1998)). Optical and near-infrared counterparts tend to be faint (e.g., $R>25$ ) making reliable redshift determinations problematic.

Star forming galaxies obey an apparently universal radio-far infrared correlation. Because the sub-mm radiation results from the same warm dust as the far infrared radiation, this correlation suggests that the radio-to-submm spectral index can be used as a redshift indicator (Carilli \& Yun(1999)), and when applied to the existing sample of faint submm objects indicates median redshifts of at least two. However at higher redshifts inverse Compton losses might quench radio emission at centimeter wavelengths. LOFAR's sensitivity is well matched to detecting these nonthermal sources at much lower rest-frame frequencies, and might be the only instrument which can utlize this technique at much higher nominal redshift $(z>5)$.

\subsection{The Galactic Distribution of Cosmic Rays}

Cosmic rays are atomic nuclei and electrons that have been accelerated to energies much greater than their rest energies and form a relativistic, collisionless, nonthermal gas pervading the interstellar medium (ISM). Their energy density is approximately $1 \mathrm{eV} \mathrm{cm}^{-3}$, meaning that cosmic rays contain at least as much energy as the other phases of the ISM.

All sky surveys at (cm-wavelength) radio and $\gamma$-ray wavelengths show similar, large-scale emission morphologies. The $\gamma$-ray emission measures the integrated product of cosmic rays and cooler ISM gas along a given line of sight while the radio data represent the same for cosmic-ray electrons and magnetic fields. Thus, the large-scale similarities could represent the cosmic ray distribution or a correlation of gas and magnetic fields. A further complicating factor is that $\mathrm{cm}$-wavelength radio emission traces $\mathrm{GeV}$ electrons while $\gamma$-ray emission traces sub-GeV electrons.

At lower frequencies $(10 \mathrm{MHz}-30 \mathrm{MHz})$, the radio synchrotron emission results from relativistic electrons with $E \approx 200-300 \mathrm{MeV}$. These electrons also radiate relativistic bremsstrahlung at the lower $\gamma$-ray photon energies. In conjuction with the Galactic $\mathrm{H} \mathrm{I}$ and $\mathrm{H}_{2}$ emission, the radio and $\gamma$-ray emission maps can be inverted to determine uniquely the distribution of cosmic-ray electrons in the Galaxy, separately from the magnetic field distribution. Furthermore, at frequencies of $10-100 \mathrm{MHz}, \mathrm{H}$ II regions become optically thick, and observations towards them can uniquely measure the synchrotron emissivity along columns with kinematically determined distances, unlike $\mathrm{cm}$ wavelength measurements which can only detect the integrated emissivity along the line of sight. This will enable the construction of a 3-D model of the Galactic cosmic-ray distribution which will offer new insights into the geometry and physics of the cosmic-ray disk and possible halo as well as potential clues about sites of cosmic-ray acceleration ((Longair(1990)).

\subsection{Solar Radar Studies}

There is at present great interest in detecting and predicting the arrival times of Earthward-directed coronal mass ejections (CMEs). CMEs that impact the Earth's magnetosphere can produce geomagnetic storms, resulting in expensive damage to spacecraft, communication, and electrical power systems. Until now the best way of studying CMEs has been from space, which is expensive, often 

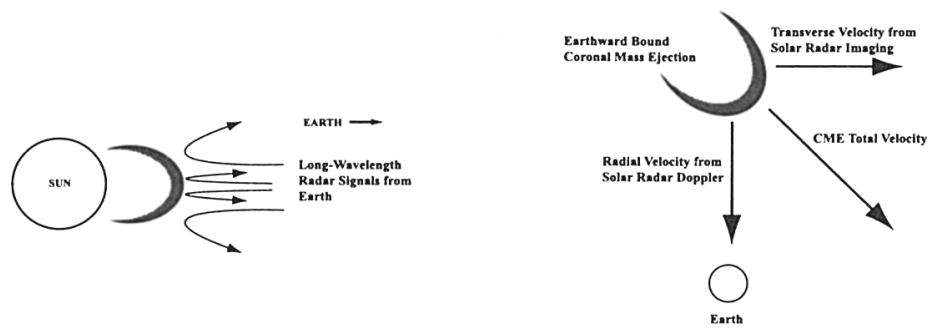

Figure 3. (Left) Geometry of solar radar. (Right) Both Dopplerdetermined radial velocities and transverse velocities acquired from imaging are required to determine accurately the Earth arrival time of a CME.

unreliable, and sub-optimal for detecting Earthward-bound CMEs. A revolutionary ground-based technique of detecting and tracking CMEs with LW solar radar is possible with LOFAR.

Figure 3 illustrates the basic principle for radar detection of CMEs. Signals transmitted from the Earth in the $20-80 \mathrm{MHz}$ range (e.g. from Arecibo) will be reflected off the solar corona and can then be imaged by LOFAR. An outward moving CME will impose a Doppler-shifted frequency and time dependent signature on this reflected signal, which can be used to image and detect it. This information could open a new window on CME studies, yielding their angular distribution, ranges, and line-of-sight velocities. Figure 3 shows how solar radar can determine the CME total velocity vector, allowing for predictions of CME arrival times.

\subsection{Coherent Emission Sources}

Bright LW emission results from coherent emission processes such as observed in the Sun, Earth, Jupiter, and pulsars. These occur readily at LWs because the size of the emitting region must be smaller than the wavelength, allowing for large regions and numbers of particles. As an example of coherently emitting LOFAR targets, we focus on pulsars. There are many other classes of coherentlyemitting objects that LOFAR could study, including extrasolar planets (Schaefer \& et al.(1998), Farrell \& et al.(1999), Dulk \& et al.(1997)).

Pulsars were discovered (Hewish \& et al.(1968)) at LWs (81 MHz), and most radio searches focus on dispersed, pulsed emission at frequencies of 400$1400 \mathrm{MHz}$. Alternate search techniques will need to be employed at LWs, as pulse broadening from scattering becomes important. At $100 \mathrm{MHz}$ only nearby pulsars - those with dispersion measures $\mathrm{DM} \leq 100 \mathrm{pc} \mathrm{cm}^{-3}$-will suffer pulse broadening less than $1 \mathrm{~s}$ and be seen as sources of pulsed emission; at $30 \mathrm{MHz}$ only a handful of the very nearest pulsars will be seen as sources of pulsed emission (Cordes(1990)).

LOFAR could serve as a useful pulsar search instrument by searching for sources having pulsar-like characteristics, other than pulsed emission. Pulsars generally, and millisecond pulsars in particular, have steep spectra (Mahoney \& 
Erickson(1985)), so compact steep-spectrum sources will be excellent candidates for deep, higher-frequency periodicity searches. A search for compact steepspectrum sources will also be less likely to select against thefollowing classes of pulsars: distant, highly scattered pulsars; sub-millisecond pulsars; and pulsars in tight binaries - objects for which there have been serious selection effects in most existing surveys.

\subsection{Surveys and Serendipitous Discoveries}

Serendipitous discoveries often provide the greatest opportunities for advances in our understanding of the physics of astronomical objects (Kellermann \& Sheets(1983)). LOFAR will be an excellent survey instrument which can be "expected" to uncover unexpected classes of objects. The array's sensitivity will allow source number counts at LWs where synchrotron aging is negligible but where cosmological tests have not yet been attempted. The capability to image a large field quickly will allow for efficient studies of variable and transient radio source populations, for example at the Galactic center (Zhao \& et al.(1992)).

\section{Low Frequency Array Concept}

Motivated by the science described above, we have prepared a series of scientific and technical requirements that will drive the design of LOFAR. Table 1 lists approximate LOFAR specifications determined to satisfy these requirements.

Table 1. LOFAR Technical Specifications

\begin{tabular}{lll}
\hline Parameter & Required & Desirable \\
\hline \hline Frequency Range & $10-150 \mathrm{MHz}$ & $3-300 \mathrm{MHz}$ \\
Collecting Area & $10^{6} \mathrm{~m}^{2}$ at $15 \mathrm{MHz}$ & $>10^{6} \mathrm{~m}^{2}$ at $15 \mathrm{MHz}$ \\
Baseline range & $100 \mathrm{~m}-150 \mathrm{~km}$ & $25 \mathrm{~m}-500 \mathrm{~km}$ \\
Angular resolution & $30^{\prime \prime}$ at $15 \mathrm{MHz}$ & $10^{\prime \prime}$ at $15 \mathrm{MHz}$ \\
& $3^{\prime \prime}$ at $150 \mathrm{MHz}$ & $1^{\prime \prime}$ at $150 \mathrm{MHz}$ \\
Sensitivity & $3 \mathrm{mJy}$ at $15 \mathrm{MHz}$ & $1 \mathrm{mJy}$ at $15 \mathrm{MHz}$ \\
& $1 \mathrm{mJy}$ at $150 \mathrm{MHz}$ & $0.3 \mathrm{mJy}$ at $150 \mathrm{MHz}$ \\
Configuration & $2 \mathrm{D}$ array, $40 \mathrm{stations}$ & $2 \mathrm{D}$ array, 100 stations \\
Spectral Resolution & $500 \mathrm{~Hz}$ & $10 \mathrm{~Hz}$ \\
Instantaneous bandwidth & $3 \mathrm{MHz}$ & $30 \mathrm{MHz}$ \\
Sky Coverage & zenith distance of $60^{\circ}$ & zenith distance of $75^{\circ}$ \\
Accessible Sky & to Galactic center & to entire inner Galaxy \\
Polarization & Full circular & Full \\
Temporal Resolution & $100 \mathrm{~ms}$ & $10 \mathrm{~ms}$ \\
independent IFs & 2 & 8 \\
Operating Philosophy & User oriented facility, Proposals solicited from the \\
& international community \\
\hline
\end{tabular}


We propose the use of electrically short active dipoles as the receiving elements. They are both frequency agile and broad-band, covering typically a decade in frequency. The dipole elements will be arranged into stations; each station is the analog of a single antenna in a system like the VLA. The stations will be approximately $160 \mathrm{~m}$ in size and contain $\approx 256$ dipoles. Many station beams can be formed and steered independently and "instantaneously" across the sky. LOFAR will have stations distributed over $500 \mathrm{~km}$ and will also have a centrally-condensed section $(\approx 30 \mathrm{~km}$ diameter $)$ that will be sensitive to extended structures, as shown in Figure 4.

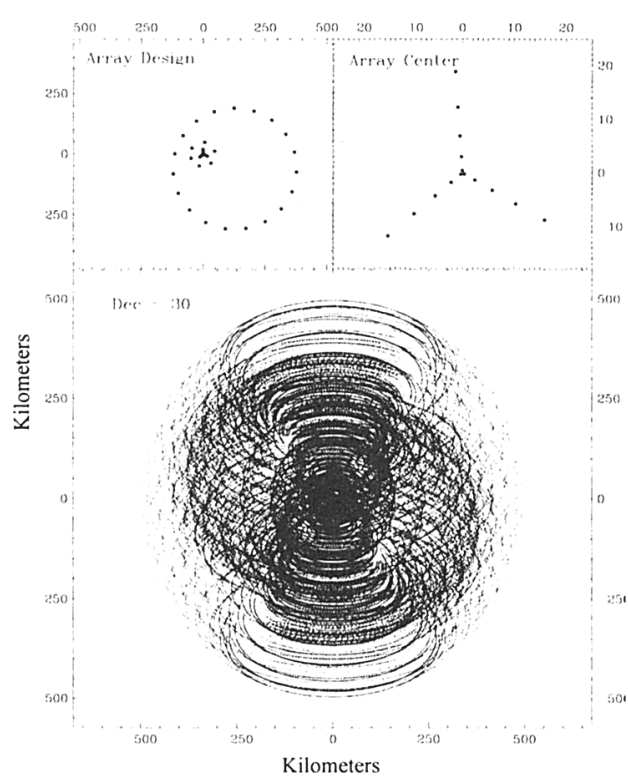

Figure 4. Possible LOFAR layout. (Top) The two top panels show the physical location of the stations. Fifteen (15) stations are arrayed in a $Y$ pattern at the center of the array. Six (6) stations surround the center in a $100 \mathrm{~km}$ (diameter) ring, and nineteen (19) stations surround these in an offset, $500 \mathrm{~km}$ (diameter) ring. (Bottom) The resulting $u-v$ coverage for a $12 \mathrm{hr}$ synthesis of a source at $\delta=30^{\circ}$.

With a total collecting area of at least $10^{6} \mathrm{~m}^{2}$ at $15 \mathrm{MHz}$, LOFAR could achieve millijansky-level sensitivity in reasonable integration times, e.g., 0.5$1 \mathrm{mJy}$ at $74 \mathrm{MHz}$ for an 8-hour integration and $3-\mathrm{MHz}$ bandwidth (Figure 5).

The NRAO VLA site in New Mexico is an example of an advantageous site for LOFAR. Existing infrastructure could be utilized, and room for inner LOFAR stations exists along the VLA arms. LOFAR's outer stations might share sites and optical fiber with the antennas in the proposed VLA expansion ("A+ configuration"). 

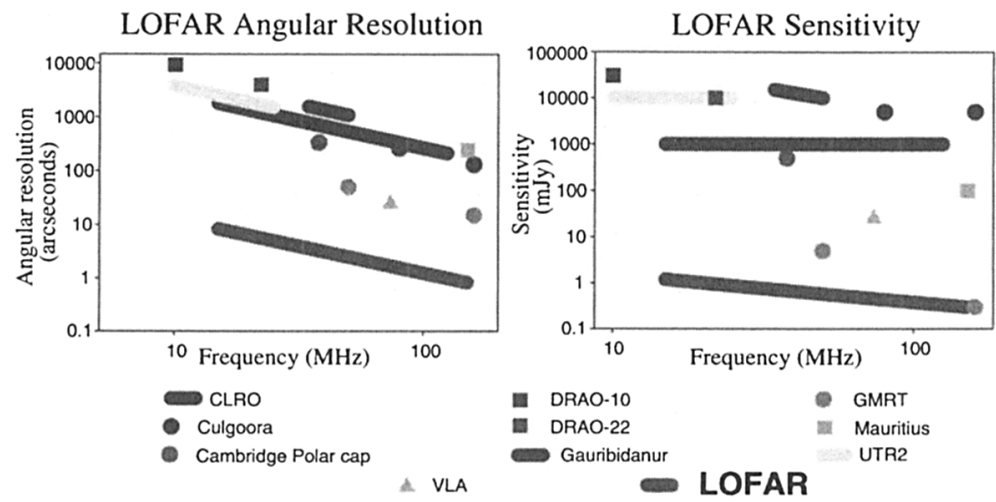

Figure 5. Resolution (arcseconds)(Left) and sensitivity (Right) vs. frequency $(\mathrm{MHz})$ for past, present, and proposed low frequency imaging instruments. LOFAR sensitivity for $10^{6} \mathrm{~m}^{2}$ at $15 \mathrm{MHz}$ collecting area( $\lambda^{2}$ dependent), $3 \mathrm{MHz}$ bandwidth, and 8 hour integration.

\section{Summary}

LOFAR will open a new, high-resolution window on the spectrum from 15$150 \mathrm{MHz}$. It will be a powerful instrument for delineating the interaction between nonthermal emitting plasmas and thermal absorbing gas, for differentiating between self-absorption processes, and for exploring the universe for coherent emission processes. For solar, planetary, Galactic, and extragalactic work it will provide unique information on the distributions of ionized gas, relativistic particles, and magnetic fields. The distribution of cosmic-rays, high-redshift radio galaxies and quasars, shocks driven by infalling matter in clusters of galaxies, pulsars in the Milky Way and in external galaxies, and radio emission from extrasolar Jovian planets are all steep-spectrum processes which could be especially well studied. It is also an ideal solar radar receiver for detecting Earth-ward bound CMEs.

Groups at the Netherlands Foundation for Research in Astronomy, MIT Haystack Observatory, NRAO, and NRL are interested in developing LOFAR, and synergetic coordination with planned expansions of existing higher-frequency facilities could enable mutual sharing of infrastructure and development costs.

Acknowledgments. We thank D. Backer, T. Bastian, K. Blundell, A. Cohen, J. Cordes, N. Duric, T. Enßlin, W. Farrell, P. Ray, L. J. Rickard, and A. Vourlidas for helpful comments. The NRAO is a facility of the National Science Foundation operated under cooperative agreement by Associated Universities, Inc. Basic research in radio astronomy at the NRL is supported by the Office of Naval Research.

\section{References}

Barger, A. J. \& et al. 1998, Nature, 394, 248 
Blundell, K. M., Rawlings, S., Eales, S. A., Taylor, G. B., \& Bradley, A. D. 1998, Mon. Not. R. Astron. Soc., 295, 265

Bowyer, S. \& Berghöfer, T. W. 1998, Astrophys. J., 506, 502, astro-ph/9804310

Carilli, C. L. \& Yun, M. S. 1999, Astrophys. J., 513, L13

Cordes, J. M. 1990, in Low Frequency Astrophysics from Space, ed. N. E. Kassim \& K. W. Weiler, Lecture Notes in Physics No. 362 (Berlin: SpringerVerlag), 165-174

Dulk, G. A., Leblanc, Y., \& Bastian, T. S. 1997, in Bull. Am. Astron. Soc., DPS meeting 29 No. 28.03 (American Astronomical Society)

Enßlin, T. A. \& Biermann, P. L. 1998, Astron.\& Astrophys., 330, 90

Farrell, W. M., Desch, M. D., \& Zarka, P. 1999, J. Geophys. Res. (Planets), 104, 14025

Hewish, A., Bell, S. J., H., Pilkington J. D. H., Scott, P. F., \& Collins, R. A. 1968, Nature, 217, 709

Hughes, D. H. \& et al. 1998, Nature, 394, 241

Hwang, C.-Y. 1997, Science, 278, 1917

Kassim, N. E. \& Erickson, W. C. 1998, in Advanced Technology MMW, Radio, and Terahertz Telescopes, Vol. 3357 (SPIE), 740-754

Kassim, N. E., Perley, R. A., Erickson, W. C., \& Dwarakanath, K. S. 1993, Astron. J., 106, 2218

Kellermann, K. \& Sheets, B., eds. 1983, Serendipitous Discoveries in Radio Astronomy, Green Bank Workshop No. 7 (National Radio Astronomy Observatory)

Kronberg, P. P. 1999, private communication

Longair, M. S. 1990, in Low Frequency Astrophysics from Space, ed. N. E. Kas$\operatorname{sim} \&$ K. W. Weiler, Lecture Notes in Physics No. 362 (Berlin: SpringerVerlag), 227-236

Madau, P. 1997, in International Astronomical Union Symposium, Vol. 186, Galaxy Interactions at Low and High Redshift, ed. D. Sanders \& J. Barnes (Dordrecht: Kluwer), 188

Mahoney, M. J. \& Erickson, W. C. 1985, Nature, 317, 154

Navarro, J., de Bruyn, A. G., Frail, D. A., Kulkarni, S. R., \& Lyne, A. G. 1995, Astrophys. J., 455, L55

Sarazin, C. L. 1999, Astrophys. J., 520, 529, astro-ph/9901061

Sarazin, C. L. \& Lieu, R. 1998, Astrophys. J., 494, L177

Schaefer, B. E., King, J. R., Deliyannis, C. P., \& Rubenstein, E. P. 1998, in Bull. Am. Astron. Soc., Vol. 193 (American Astronomical Society)

Schlickeiser, R., Sievers, A., \& Thiemann, H. 1987, Astron.\& Astrophys., 182, 21

Smail, I., Ivison, R. J., \& Blain, A. W. 1997, Astrophys. J., 490, L5

Zhao, J.-H. \& et al. 1992, Science, 255, 1538 